\title{
Accuracy Analysis for the Solution of Initial Value Problem of ODEs Using Modified Euler Method
}

\author{
Mohammad Asif Arefin* \\ Department of Mathematics, Jashore University of Science and Technology, Jashore-7408, Bangladesh \\ *Corresponding Author: Mohammad Asif Arefin (asif.math@just.edu.bd, ORCiD: 0000-0002-2892-1683) \\ Email: asif.math@just.edu.bd
}

\section{Nazrul Islam}

Department of Mathematics, Jashore University of Science and Technology, Jashore-7408, Bangladesh

Email: nazrul.math@just.edu.bd

\author{
Biswajit Gain \\ Department of Mathematics, Jashore University of Science and Technology, Jashore-7408, Bangladesh \\ Email: biswajitmath25@gmail.com
}

\author{
Md. Roknujjaman \\ Department of Engineering Mechanic and Energy, University of Tsukuba. Japan \\ Email: rkripon.mathiu@gmail.com
}

Received: 14 November 2020; Accepted: 10 January 2021; Published: 08 June 2021

\begin{abstract}
There exist numerous numerical methods for solving the initial value problems of ordinary differential equations. The accuracy level and computational time are not the same for all of these methods. In this article, the Modified Euler method has been discussed for solving and finding the accurate solution of Ordinary Differential Equations using different step sizes. Approximate Results obtained by different step sizes are shown using the result analysis table. Some problems are solved by the proposed method then approximated results are shown graphically compare to the exact solution for a better understanding of the accuracy level of this method. Errors are estimated for each step and are represented graphically using Matlab Programming Language and MS Excel, which reveals that so much small step size gives better accuracy with less computational error. It is observed that this method is suitable for obtaining the accurate solution of ODEs when the taken step sizes are too much small.
\end{abstract}

Index Terms: Modified Euler method; Initial Value Problems; Estimation of Error; and Accuracy Analysis.

\section{Introduction}

In the field of science, engineering, and technology every day numerous problems are arises related to the differential equation which has the form of either ordinary differential equations or partial differential equations. Though there are many methods to solve ordinary differential equations, sometimes it is tough or quite impossible to solve IVPs of the ordinary differential equation, analytically. In those cases, the numerical approximation is used for solving these kinds of problems numerically. There exist various numerical methods, where the accuracy level and computational time are not the same for all of these numerical methods.

From the literature review analysis, we can comprehend that numerous writers have taken a shot at numerical solutions of ordinary differential equations utilizing various numerical methods, for example, Runge-Kutta fourth-order method, Euler's method, Modified Euler's method and, and so forth. Numerous authors have stepped up, to keep up the high accuracy for the solution of IVPs of ODEs. M. A. Islam [1] attempts to discover accurate solutions by utilizing the RK4 technique for solving IVPs. At the same time, M. A. Islam [2] represents the accuracy analysis of IVPs of ODEs with the help of the Euler method. L. F. Shampine, H. A. Watts, and M. Babul Hossain have compared the error for the RK4 method in [3], [4]. A. B. M. Hame et al. [5] discussed the accuracy of Euler and modified Euler technique. Detailed explanation and higher-level applications can be found in [6]-[10] about IVPs of ODEs where the Modified 
Euler method is used. N. Samsudin et al. explain the uses of the modified Euler method in the case of cube arithmetic [11] .In this research, Modified Euler method has been applied to solve the IVPs of ODEs. Here, Different step sizes have been used for finding the best approximation. In each step, approximate solutions are compared to the exact solutions graphically using MATLAB programming language.

The rest of the segment of the article is structured as: In segment 2, we have presented the formulation of the method. in segment 3, some numerical examples are solved using the proposed method. In segment 4 , we have discussed our finding results elaborately. In the lattermost part, the conclusion is given.

\section{Formation of Method}

In the case of the Euler method, the slope is calculated for the given step size, and the tangent is drawn at a point. Consequently, for linear functions, the performance of this technique is best, however for different cases, truncation error has occurred. To take care of this issue the Modified Euler method is inaugurated. For the solution of IVPs with the best credibility and accuracy, the Modified Euler method plays a vital role. It solves initial value problems of ordinary differential equations (ODE) by approximating in an interval with slope as an arithmetic average. This strategy is a facile enhancement for Euler's method in function evaluation per step but leads to yield a second-order method.

In this strategy rather than a point, the arithmetic average of the slope over an interval $\left(x_{n}, x_{n+1}\right)$ is used. So, in the Modified Euler method for each step the predicted value of $y_{n+1}$ is calculated first, by using the Euler's method and then the slopes at the points $\left(x_{n}, y_{n}\right)$ and $\left(x_{n+1}, y_{n+1}\right)$ is calculated and the arithmetic average of these slopes are added to $y_{n}$, so that we can calculate the corrected value of $y_{n+1}$

So, initially the value is predicted for a step $(n+1$, is taken here):

$$
y_{n+1, p}=y_{n}+h * f\left(x_{n}, y_{n}\right)
$$

Here $\mathrm{h}$ stands for the step size for each increment

Finally, the predicted value is corrected as

$$
y_{n+1, c}=y_{n}+h * \frac{f\left(x_{n}, y_{n}\right)+f\left(x_{n+1}, y_{n+1, p}\right)}{2}
$$

The incrimination is done by $x_{n+1}=x_{n}+h, n=n+1$

Since, in this strategy, the average slope is utilized, so the amount of error is decreased essentially. Likewise, the process of correction for convergence can be repeated gradually. Thus at every step, we can reduce the error by improving the value of $y$

\section{Numerical Examples Solved by the Proposed Method}

In this section, three IVPs of ODEs are taken into account to verify the accuracy of the proposed method. The numerical approximation, as well as errors, is calculated for different step sizes by the proposed method using the MATLAB programming language. Exact solutions have also been calculated with the approximate solution for all three examples, which reveals the accuracy level of the proposed method. Finally, exact solutions and approximate solutions are represented graphically using MATLAB programming language. Error analysis is also introduced graphically where MATLAB and MS Excel are used.

Example-1. Taking the initial value problem $y^{\prime}=1+(x-y)^{2}, y(2)=1$ on the interval $2 \leq x \leq 3$. Exact solution of the problem is $y(x)=x+\frac{1}{1-x} \cdot[12]$

Table. 1(a) represents exact solution along with the approximate solutions of different step sizes obtained by the Modified Euler method. Table. 1(b) represents the error estimation for different step sizes.

The curves of the exact and approximate solutions are displayed in Fig.:1(a)-(e) for different step size. The error estimation for different step sizes are shown in Fig. 2 and Fig. 3 
Table. 1 (a): Approximate solution for different step sizes with exact solution

\begin{tabular}{|c|c|c|c|c|c|}
\hline \multirow[t]{2}{*}{$\mathbf{x}$} & \multicolumn{4}{|c|}{ Approximate solution } & \multirow{2}{*}{ Exact } \\
\hline & $\mathrm{h}=\mathbf{0 . 1}$ & $h=0.05$ & $h=0.025$ & $h=0.0125$ & \\
\hline 2.00 & 1.000000000000000 & 1.000000000000000 & 1.000000000000000 & 1.000000000000000 & 1.000000000000000 \\
\hline 2.10 & 1.190500000000000 & 1.190810811991210 & 1.190885058416040 & 1.190903151596080 & 1.190909090909090 \\
\hline 2.20 & 1.366037851531090 & 1.366515499412070 & 1.366629676278950 & 1.366657521241190 & 1.366666666666660 \\
\hline 2.30 & 1.530028845961610 & 1.530591121841590 & 1.530725621962890 & 1.530758445232020 & 1.530769230769230 \\
\hline 2.40 & 1.684925325698840 & 1.685524367469300 & 1.685667761099870 & 1.685702775517610 & 1.685714285714280 \\
\hline 2.50 & 1.832532830650470 & 1.833140517038160 & 1.833286076758060 & 1.833321638928020 & 1.833333333333330 \\
\hline 2.60 & 1.974209664372280 & 1.974809521569860 & 1.974953296939430 & 1.974988439846600 & 1.975000000000000 \\
\hline 2.70 & 2.110997019660380 & 2.111579585149490 & 2.111719299453880 & 2.111753464321390 & 2.111764705882350 \\
\hline 2.80 & 2.243706253344960 & 2.244266345469180 & 2.244400745522800 & 2.244433623592380 & 2.244444444444440 \\
\hline 2.90 & 2.372978888363630 & 2.373513960766600 & 2.373642424678460 & 2.373673861647850 & 2.373684210526310 \\
\hline 3.00 & 2.499328778717240 & 2.499837909669030 & 2.499960205652050 & 2.499990142839880 & 2.500000000000000 \\
\hline
\end{tabular}

Table. 1 (b): Approximate error estimation for different step sizes

\begin{tabular}{ccccc}
\hline $\mathbf{X}$ & $\mathbf{h}=\mathbf{0 . 1}$ & $\mathbf{h}=\mathbf{0 . 0 5}$ & $\mathbf{h}=\mathbf{0 . 0 2 5}$ & $\mathbf{h}=\mathbf{0 . 0 1 2 5}$ \\
\cline { 2 - 5 } & 0.000000000000000 & 0.000000000000000 & 0.000000000000000 \\
\hline 2.00 & 0.000409090909091 & 0.000098278917876 & 0.000024032493048 & 0.000000000000000 \\
2.10 & 0.000628815135569 & 0.000151167254596 & 0.000036990387711 & 0.000005939313005 \\
2.30 & 0.000740384807619 & 0.000178108927636 & 0.000043608806339 & 0.000009145425475 \\
2.40 & 0.000788960015442 & 0.000189918244977 & 0.000046524614405 & 0.000010785537209 \\
2.50 & 0.000800502682858 & 0.000192816295171 & 0.000047256575264 & 0.000011510196680 \\
2.60 & 0.000790335627717 & 0.000190478430132 & 0.000046703060560 & 0.000011694405322 \\
2.70 & 0.000767686221973 & 0.000185120732853 & 0.000045406428460 & 0.000011560153406 \\
2.80 & 0.000738191099484 & 0.000178098975253 & 0.000043698921632 & 0.000011241560977 \\
2.90 & 0.000705322162682 & 0.000170249759704 & 0.000041785847846 & 0.000010820852077 \\
3.00 & 0.000671221282755 & 0.000162090330964 & 0.000039794347942 & 0.000009857160136 \\
\hline
\end{tabular}

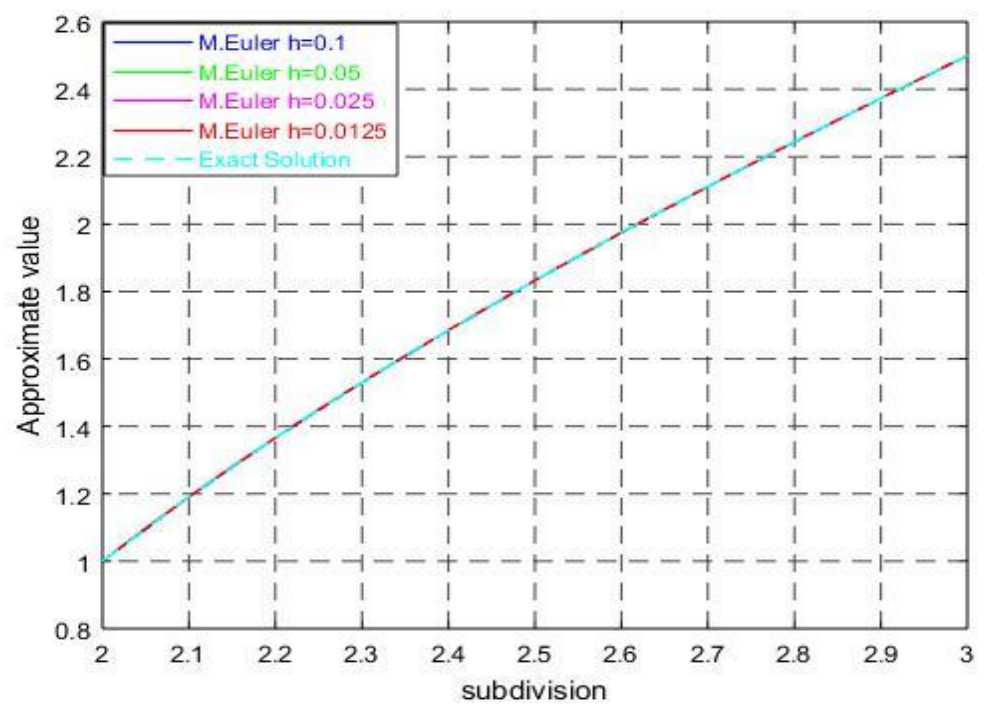

Fig. 1(a). Exact numerical solutions curve. 


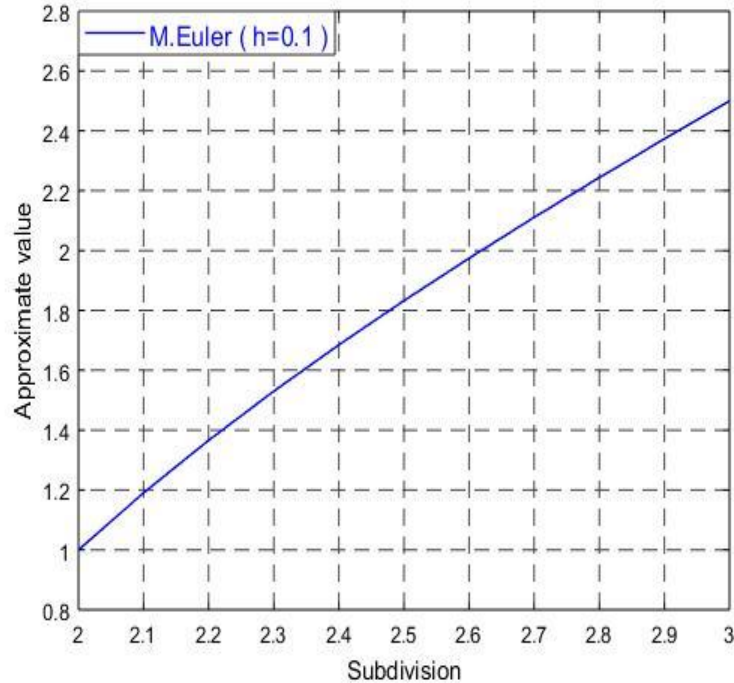

Fig. 1(b). Approximate curve for step size $h=0.1$

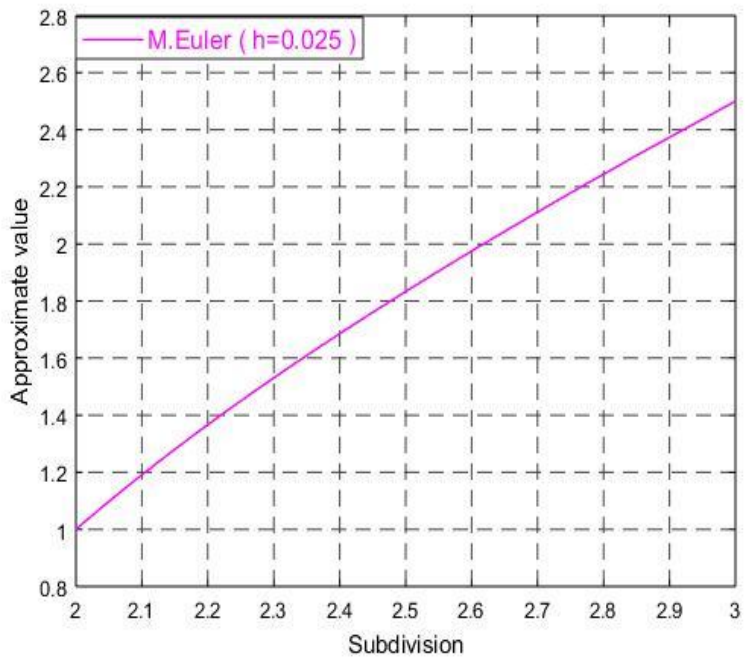

Fig. 1(d). Approximate curve for step size $h=0.025$

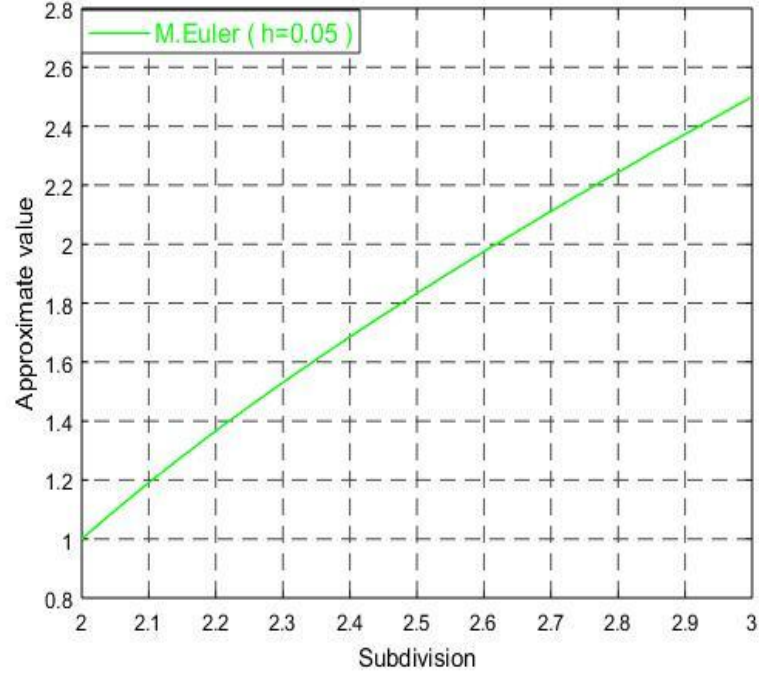

Fig. 1(c). Approximate curve for step size $\mathrm{h}=0.05$

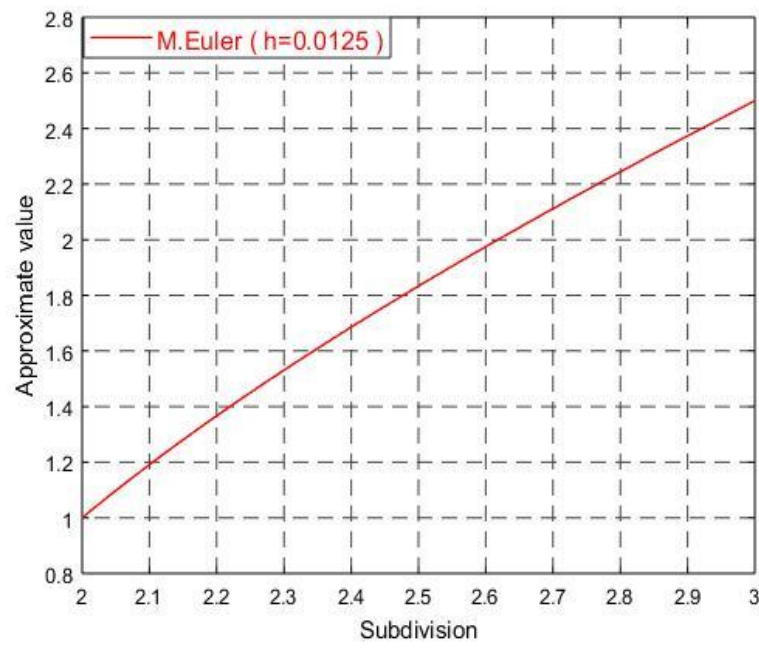

Fig. 1(e). Approximate curve for step size $h=0.0125$

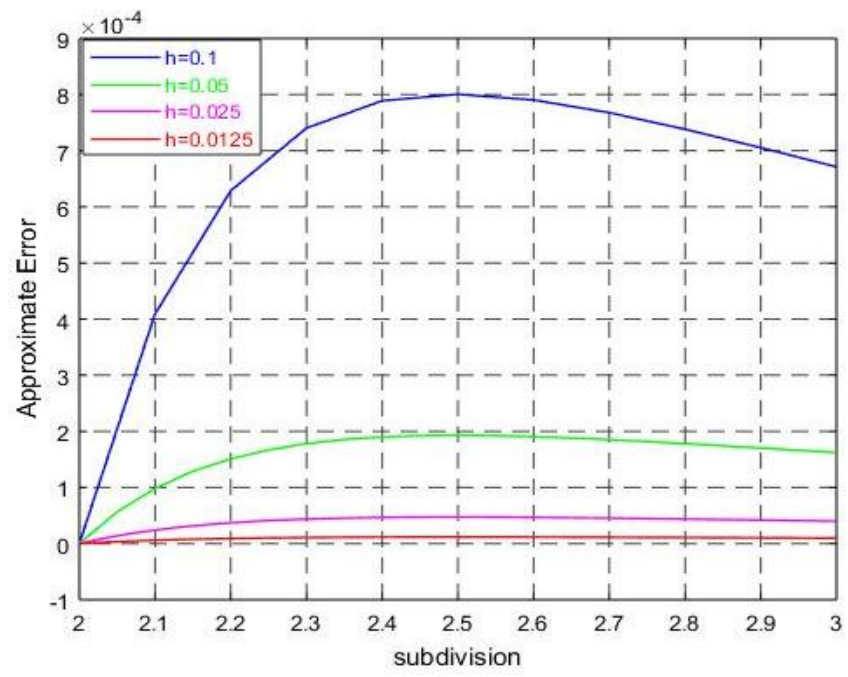

Fig. 2. Error estimation for different step size obtained by Modified Euler method using Matlab 


\section{Error Comparison}

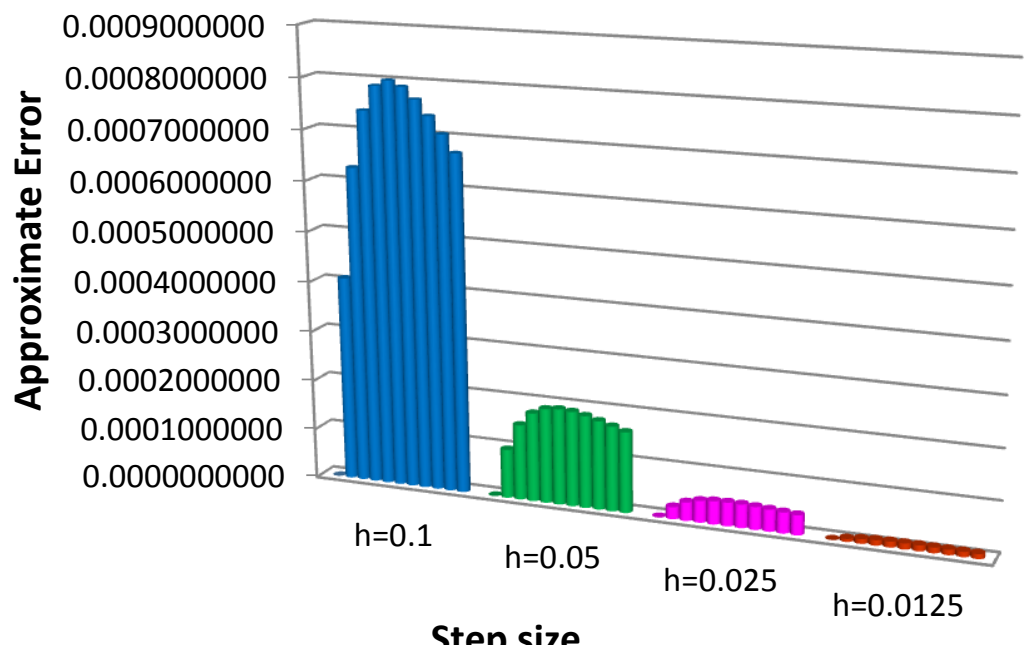

Step size

Fig. 3. Error estimation for different step size obtained by Modified Euler method using MS Excel

Example-2. Taking the initial value problem $y^{\prime}=\frac{1+x}{1+y}, y(1)=2$ on the interval $1 \leq x \leq 2$. Exact solution of the problem is $y(x)=\sqrt{\left(x^{2}+2 x+6\right)-1} \cdot[12]$

Table: 2(a) represents exact solution along with the approximate solutions of different step sizes obtained by the Modified Euler method. Table: 2(b) represents the error estimation for different step sizes.

The curves of the exact and approximate solutions are displayed in Fig.: 4(a)-(e) for different step size. The error estimation for different step sizes are shown in Fig. 5 and Fig. 6

Table. 2 (a): Approximate solution for different step sizes with exact solution

\begin{tabular}{|c|c|c|c|c|c|}
\hline \multirow{2}{*}{$\mathbf{x}$} & \multicolumn{4}{|c|}{ Approximate solution } & \multirow{2}{*}{ Exact } \\
\hline & $\mathrm{h}=\mathbf{0 . 1}$ & $\mathrm{h}=\mathbf{0 . 0 5}$ & $h=0.025$ & $\mathrm{~h}=\mathbf{0 . 0 1 2 5}$ & \\
\hline 1.00 & 2.000000000000000 & 2.000000000000000 & 2.000000000000000 & 2.000000000000000 & 2.000000000000000 \\
\hline 1.10 & 2.067572463768110 & 2.067572346569500 & 2.067572332090930 & 2.067572330291790 & 2.067572330035590 \\
\hline 1.20 & 2.136877673445990 & 2.136877458583370 & 2.136877432039670 & 2.136877428741320 & 2.136877428271620 \\
\hline 1.30 & 2.207803324047900 & 2.207803028205150 & 2.207802991657280 & 2.207802987115810 & 2.207802986469080 \\
\hline 1.40 & 2.280244307151070 & 2.280243944528720 & 2.280243899730710 & 2.280243894164050 & 2.280243893371340 \\
\hline 1.50 & 2.354102442478850 & 2.354102025128570 & 2.354101973568930 & 2.354101967162050 & 2.354101966249680 \\
\hline 1.60 & 2.429286166933670 & 2.429285705057910 & 2.429285647996730 & 2.429285640906170 & 2.429285639896440 \\
\hline 1.70 & 2.505710196603090 & 2.505709698820170 & 2.505709637321860 & 2.505709629679870 & 2.505709628591610 \\
\hline 1.80 & 2.583295174128530 & 2.583294647704610 & 2.583294582666600 & 2.583294574584690 & 2.583294573433780 \\
\hline 1.90 & 2.661967311120090 & 2.661966762170670 & 2.661966694348290 & 2.661966685920310 & 2.661966684720100 \\
\hline 2.00 & 2.741658033015760 & 2.741657466679430 & 2.741657396707400 & 2.741657388012200 & 2.741657386773930 \\
\hline
\end{tabular}


Table. 2 (b): Approximate error estimation for different step sizes

\begin{tabular}{ccccc}
\hline $\mathbf{x}$ & $\mathbf{h}=\mathbf{0 . 1}$ & $\mathbf{h}=\mathbf{0 . 0 5}$ & $\mathbf{h}=\mathbf{0 . 0 2 5}$ & $\mathbf{h}=\mathbf{0 . 0 1 2 5}$ \\
\cline { 2 - 5 } & 0.000000000000000 & 0.000000000000000 & 0.000000000000000 \\
1.00 & 0.000000133732522 & 0.000000016533908 & 0.000000002055340 & 0.000000000000000 \\
1.10 & 0.000000245174375 & 0.000000030311755 & 0.000000003768051 & 0.000000000256206 \\
1.30 & 0.000000337578816 & 0.000000041736071 & 0.000000005188198 & 0.000000000469700 \\
1.40 & 0.000000413779725 & 0.000000051157377 & 0.000000006359366 & 0.000000000646724 \\
1.50 & 0.000000476229173 & 0.000000058878887 & 0.000000007319252 & 0.000000000792714 \\
1.60 & 0.000000527037222 & 0.000000065161466 & 0.000000008730243 & 0.000000001009729 \\
1.70 & 0.000000568011473 & 0.000000070228551 & 0.000000009232821 & 0.000000001088260 \\
1.80 & 0.000000600694749 & 0.000000074270830 & 0.000000009628188 & 0.000000001150912 \\
1.90 & 0.000000626399985 & 0.000000077450561 & 0.000000009933463 & 0.000000001200202 \\
2.00 & 0.000000646241828 & 0.000000079905490 & 0.000000001238262 & \\
\hline
\end{tabular}

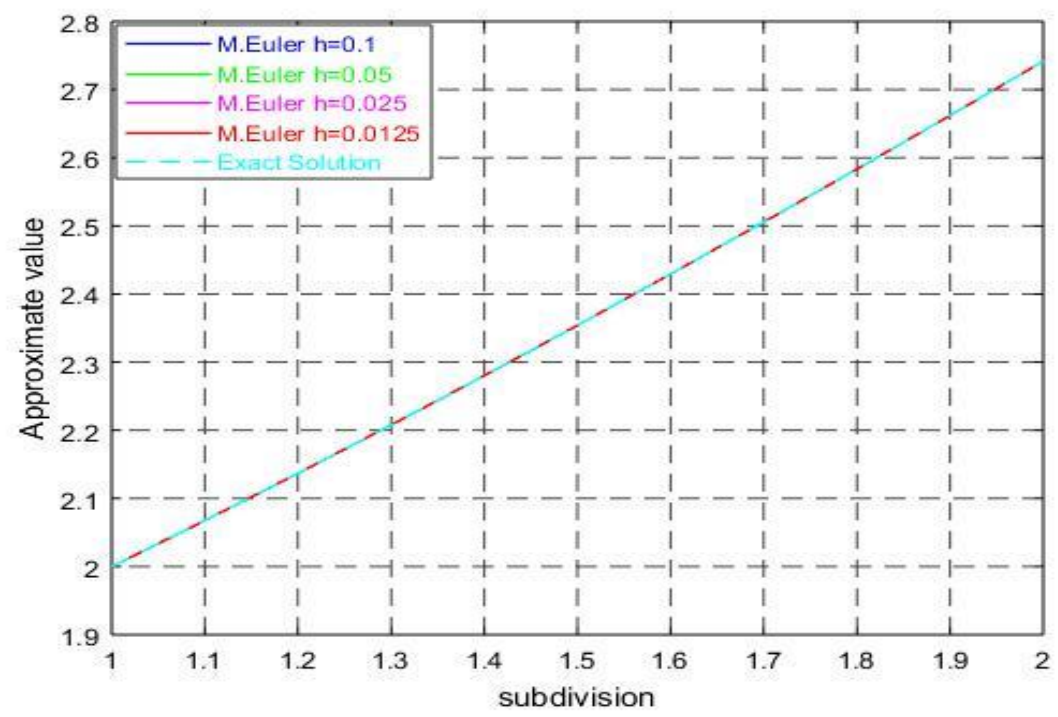

Fig. 4(a). Exact numerical solutions curve.

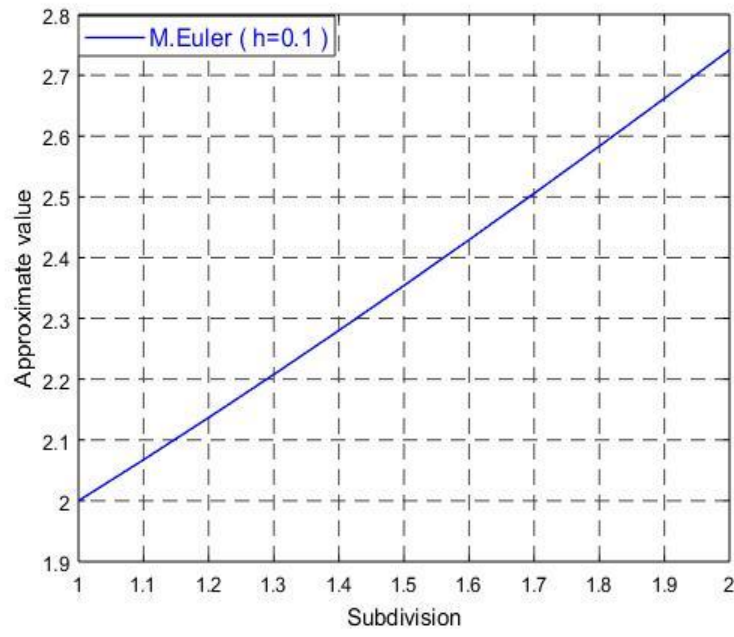

Fig. 4(b). Approximate curve for step size $h=0.1$

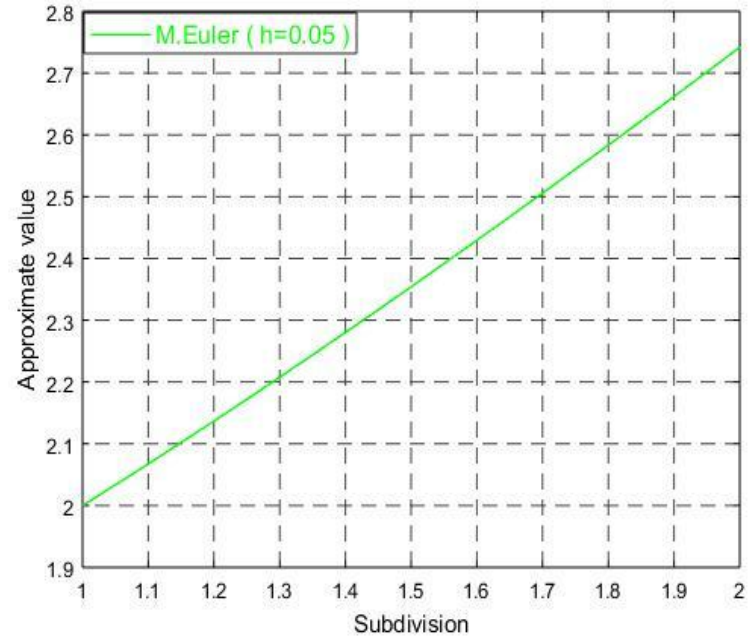

Fig. 4(c). Approximate curve for step size $h=0.05$ 


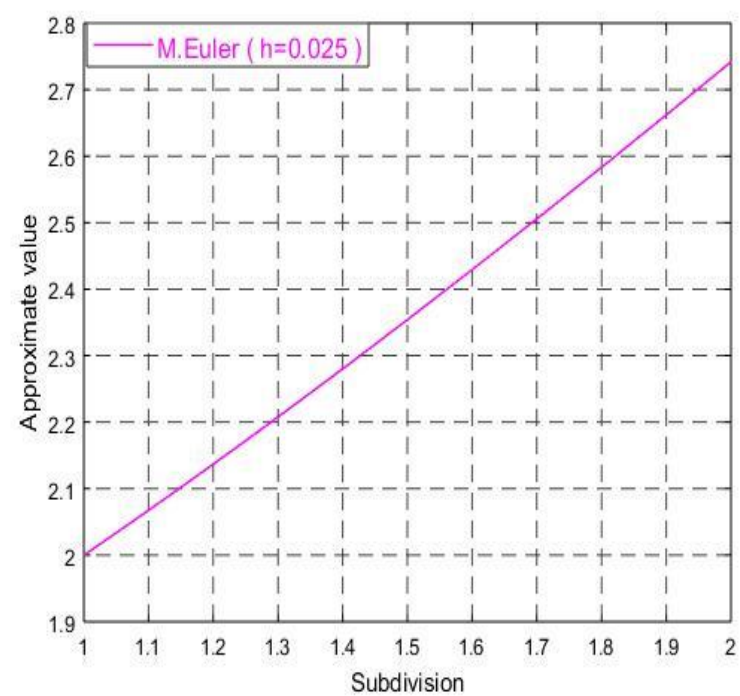

Fig. 4(d). Approximate curve for step size $\mathrm{h}=0.025$

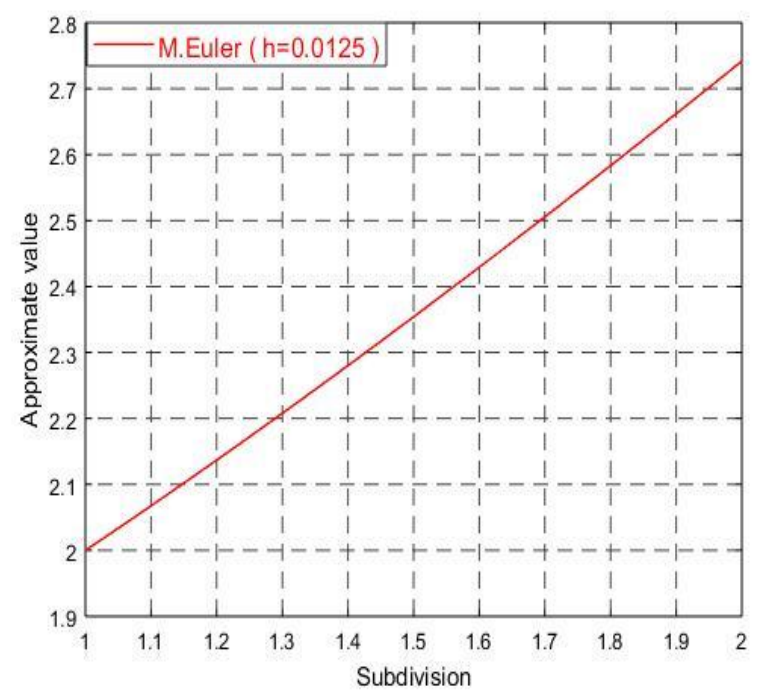

Fig. 4(e). Approximate curve for step size $\mathrm{h}=0.0125$

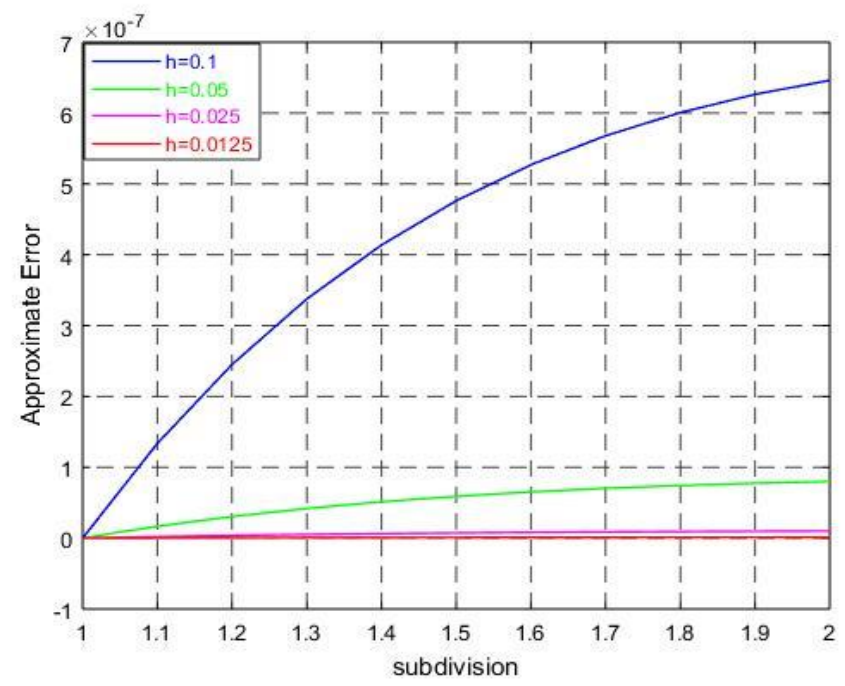

Fig. 5. Error estimation for different step size obtained by Modified Euler method using Matlab

\section{Error Comparison}

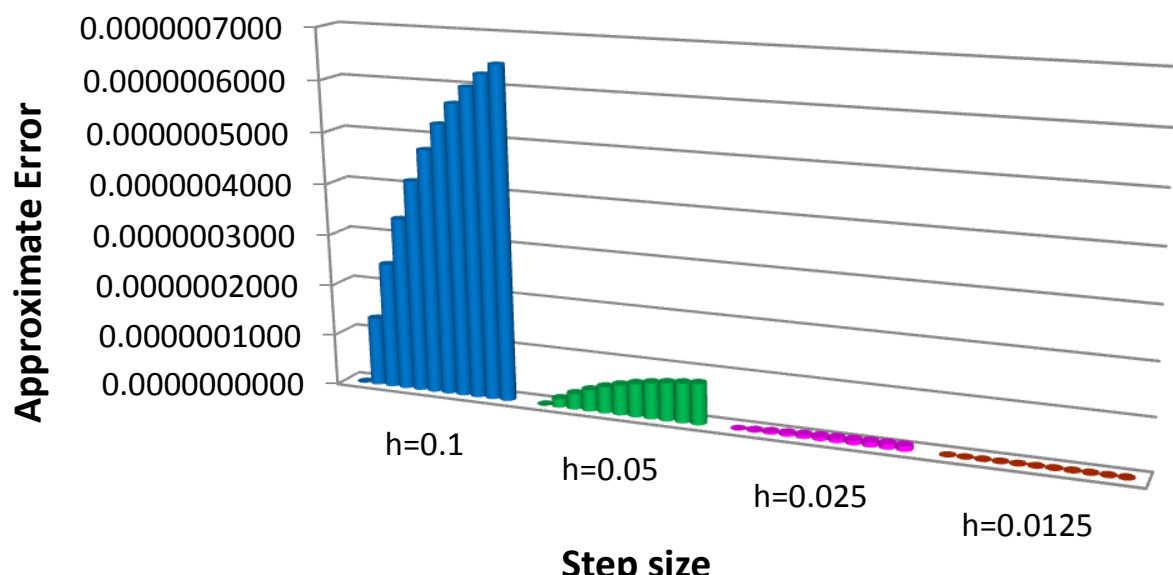

Step size

Fig. 6. Error estimation for different step size obtained by Modified Euler method using MS Excel 
Example-3. Taking the initial value problem $y^{\prime}=-x y+\frac{4 x}{y}, y(0)=1$ on the interval $0 \leq x \leq 1$. Exact solution of the problem is $y(x)=\sqrt{\left(4-3 e^{-x^{2}}\right)}$.

Table: 3(a) represents exact solution along with the approximate solutions of different step sizes obtained by the Modified Euler method. Table: 3(b) represents the error estimation for different step sizes.

The curves of the exact and approximate solutions are displayed in Fig. :7(a)-(e) for different step size. The error estimation for different step sizes are shown in Fig. 8 and Fig. 9

Table. 3 (a): Approximate solution for different step sizes with exact solution

\begin{tabular}{|c|c|c|c|c|c|}
\hline \multirow[b]{2}{*}{$\mathbf{x}$} & \multicolumn{4}{|c|}{ Approximate solution } & \multirow[b]{2}{*}{ Exact } \\
\hline & $\mathrm{h}=0.1$ & $h=0.05$ & $h=0.025$ & $\mathrm{~h}=\mathbf{0 . 0 1 2 5}$ & \\
\hline 0.00 & 1.000000000000000 & 1.000000000000000 & 1.000000000000000 & 1.000000000000000 & 1.000000000000000 \\
\hline 0.10 & 1.014999999999990 & 1.014837832961770 & 1.014818216463310 & 1.014815825826070 & 1.014815499858220 \\
\hline 0.20 & 1.057491523163810 & 1.057215644198840 & 1.057184571579510 & 1.057181276299050 & 1.057181007464200 \\
\hline 0.30 & 1.122019286443720 & 1.121724352800180 & 1.121698286656330 & 1.121697319563580 & 1.121698018267980 \\
\hline 0.40 & 1.201686153997620 & 1.201479132995020 & 1.201477705849140 & 1.201483140320270 & 1.201486010364400 \\
\hline 0.50 & 1.289770700715860 & 1.289742952021200 & 1.289783551824630 & 1.289799108565990 & 1.289805276305600 \\
\hline 0.60 & 1.380583708338410 & 1.380798062290510 & 1.380892698939980 & 1.380920963253040 & 1.380931215443730 \\
\hline 0.70 & 1.469715677117440 & 1.470204034701250 & 1.470358372013860 & 1.470400506202230 & 1.470415185397220 \\
\hline 0.80 & 1.553976920775490 & 1.554742949367590 & 1.554956629561170 & 1.555012413068220 & 1.555031423435180 \\
\hline 0.90 & 1.631230850967540 & 1.632254692221930 & 1.632522274622570 & 1.632590310720120 & 1.632613181782860 \\
\hline 1.00 & 1.700210295378890 & 1.701453936551950 & 1.701766083795120 & 1.701844077150750 & 1.701870052761270 \\
\hline
\end{tabular}

Table. 3 (b): Approximate error estimation for different step sizes

\begin{tabular}{lcccc}
\hline & & \multicolumn{3}{c}{ Errors } \\
\cline { 2 - 5 } $\mathbf{x}$ & $\mathbf{h = 0 . 1}$ & $\mathbf{h = 0 . 0 5}$ & $\mathbf{h = 0 . 0 2 5}$ & $\mathbf{h = 0 . 0 1 2 5}$ \\
\hline 0.00 & 0.000000000000000 & 0.000000000000000 & 0.000000000000000 & 0.000000000000000 \\
0.10 & 0.000184500141776 & 0.000022333103553 & 0.000002716605091 & 0.000000325967853 \\
0.20 & 0.000310515699610 & 0.000034636734637 & 0.000003564115308 & 0.000000268834848 \\
0.30 & 0.000321268175742 & 0.000026334532200 & 0.000000268388356 & 0.000000698704396 \\
0.40 & 0.000200143633225 & 0.000006877369376 & 0.000008304515258 & 0.000006167739608 \\
0.50 & 0.000034575589746 & 0.000062324284400 & 0.000021724480976 & 0.000010252190692 \\
0.60 & 0.000347507105315 & 0.000133153153223 & 0.000038516503745 & 0.000014679194990 \\
0.70 & 0.000699508279775 & 0.000211150695967 & 0.000056813383360 & 0.000019010366955 \\
0.80 & 0.001054502659688 & 0.000288474067590 & 0.000074793874013 & 0.000022871062744 \\
0.90 & 0.001382330815323 & 0.000358489560934 & 0.000090907160295 & 0.000025975610518 \\
1.00 & 0.001659757382382 & 0.000416116209326 & 0.000103968966150 & \\
\hline
\end{tabular}

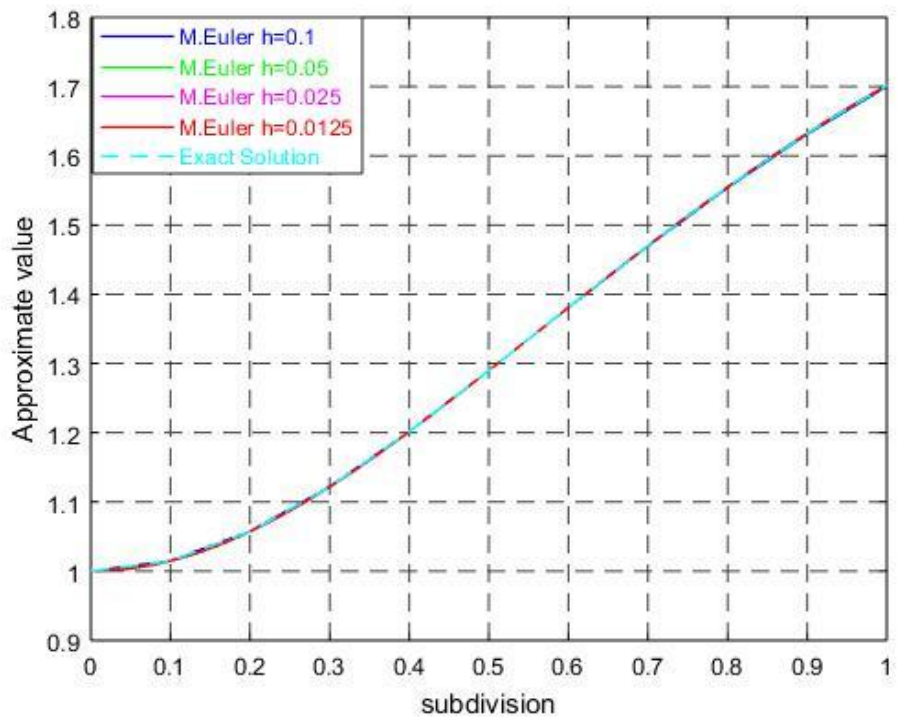

Fig. 7(a). Exact numerical solutions curve 


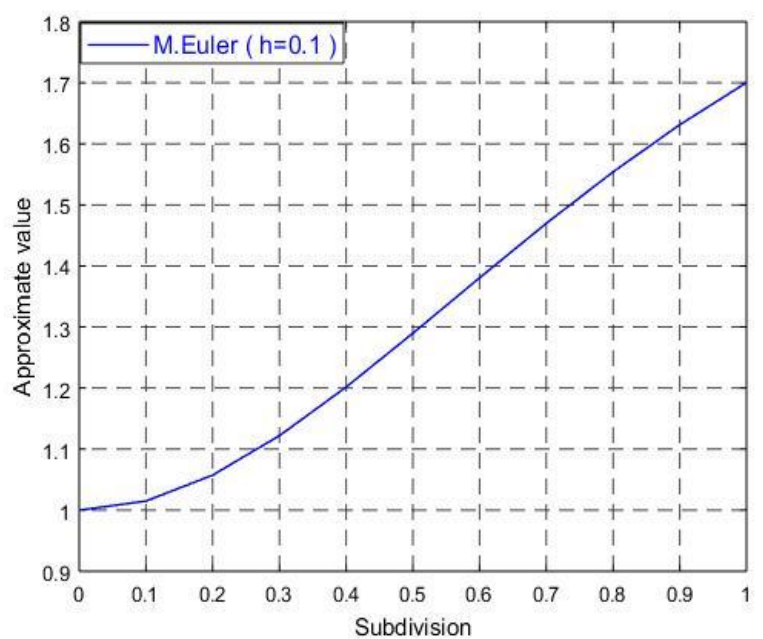

Fig. 7(b). Approximate curve for step size $h=0.1$

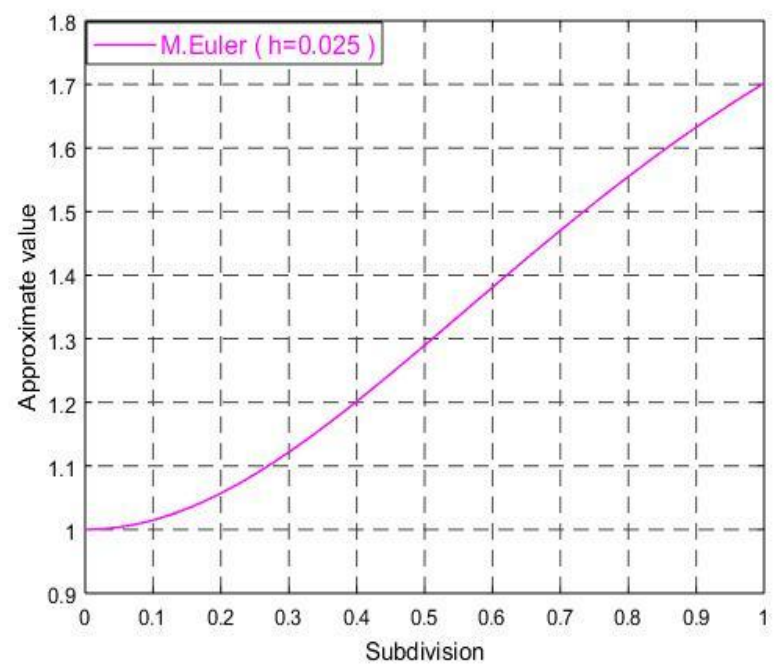

Fig. 7(d). Approximate curve for step size $\mathrm{h}=0.025$

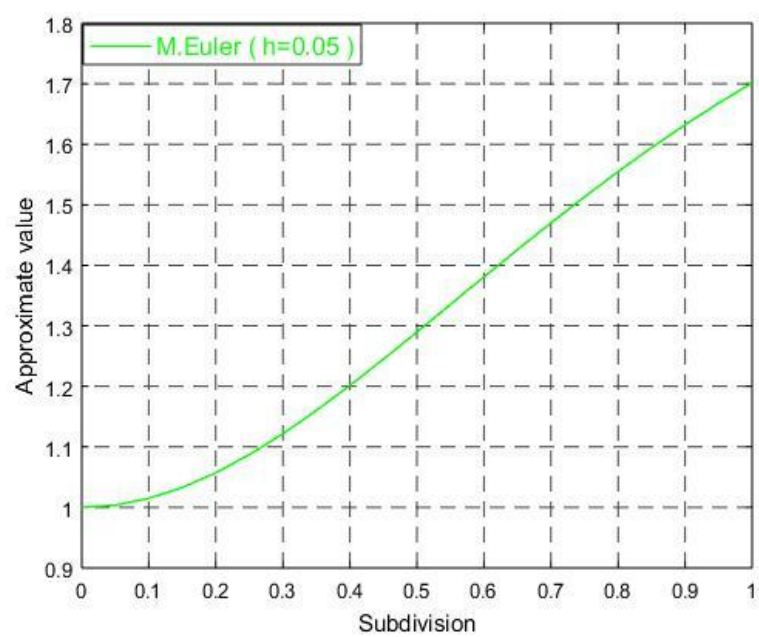

Fig. 7(c). Approximate curve for step size $\mathrm{h}=0.05$

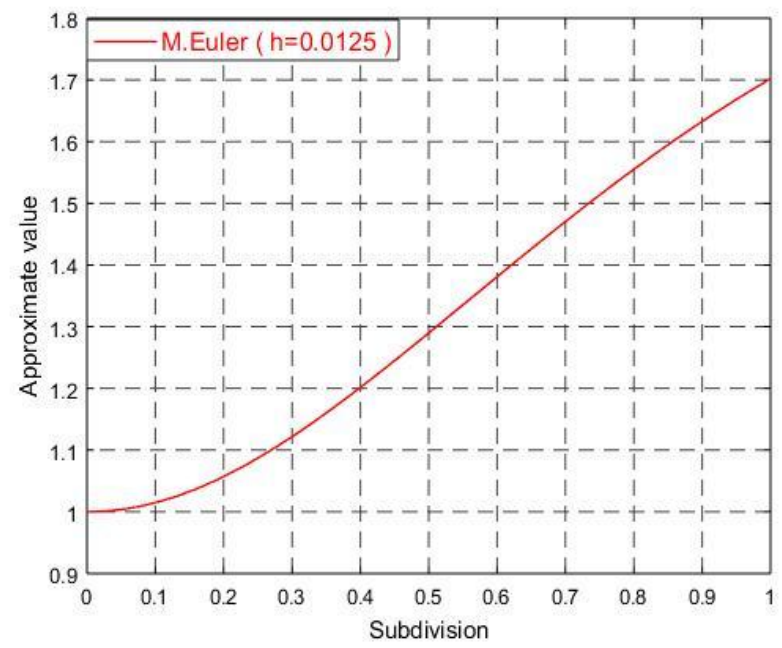

Fig. 7(e). Approximate curve for step size $\mathrm{h}=0.0125$

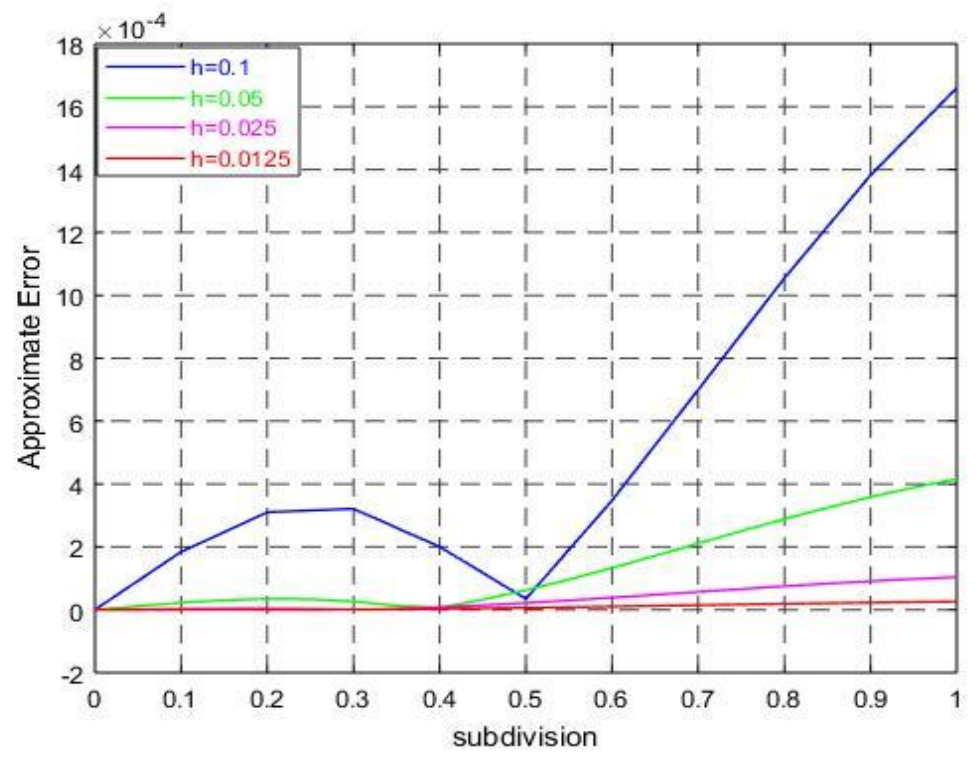

Fig. 8. Error estimation for different step size obtained by Modified Euler method using Matlab 


\section{Error Comparison}

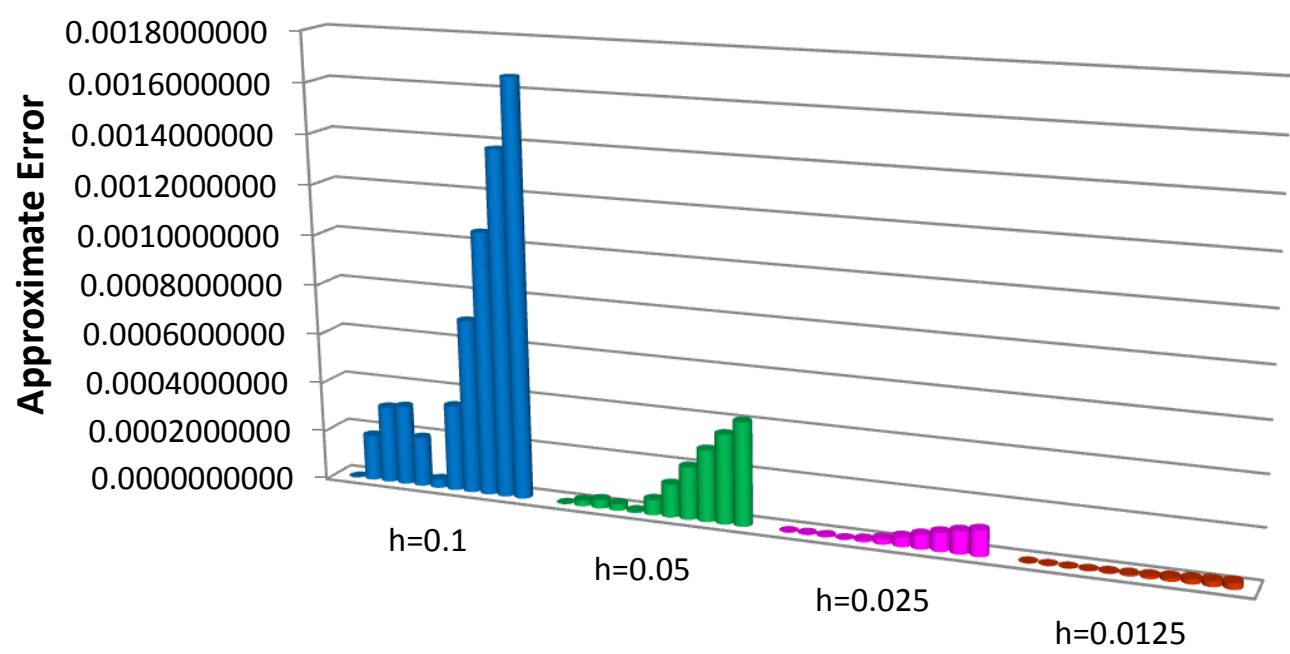

Step size

Fig. 9. Error estimation for different step size obtained by Modified Euler method using MS Excel

\section{Discussion of Results}

The exact solution along with approximate solutions with different step sizes for the taken three IVPs is represented by Table: 1(a), 2(a), and 3(a). The error estimation with different step sizes for the taken three IVPs is represented by Table: 1(b), 2(b), and 3(b). For all cases, it has been observed that the convergences rate of approximate solutions to exact solutions is not so high but the amount of error is high when the considering step size is comparatively large. On the other hand, it has been observed that the convergences rate of approximate solutions to exact solutions is high when the considering step size is too small. The above statements are also validated sharply by the error estimation (Figures:2-3, Figures:5-6, and Figures:8-9) graphs. In nutshell, it has been observed that we can reach up to the accurate solutions of IVPs of ODEs by utilizing the Modified Euler method when the step size is so much small.

\section{Conclusion}

In this article, the Modified Euler method has been discussed for finding the accurate solution of IVPs of ordinary differential equations. The numerical approximations of each taken example obtained by the Modified Euler method are best fitted with the exact solution. Result tables and error tables reveal that the accuracy level of the proposed method slightly varies for different step sizes whereas, in all cases, accurate numerical approximation comes from the least step size. It has been noted that for each step, the convergence rate for the approximate solution to the exact solution is good, whereas, for the minimum step size, the proposed method gives the best approximation result. Consequently, too much small step size must be taken to achieve the precise solution using the Modified Euler method. In the field of science and engineering, the proposed method may play a vital role in solving ordinary differential equations.

\section{Conflict of Interests}

Authors guarantee that in this article, none of the authors have any contest of interests.

\section{References}

[1] M. A. Islam, "Accuracy Analysis of Numerical solutions of initial value problems (IVP) for ordinary differential equations (ODE)," IOSR J. Math. Ver. III, vol. 11, no. 3, pp. 2278-5728, 2015, doi: 10.9790/5728-11331823.

[2] M. A. Islam, "Accurate Solutions of Initial Value Problems for Ordinary Differential Equations with the Fourth Order Runge Kutta Method," J. Math. Res., vol. 7, no. 3, pp. 41-45, 2015, doi: 10.5539/jmr.v7n3p41.

[3] L. F. Shampine and H. A. Watts, "Comparing Error Estimators for Runge-Kutta Methods," Math. Comput., vol. 25, no. 115, p. 445, 1971, doi: 10.2307/2005206.

[4] M. Babul Hossain, “A Comparative Study on Fourth Order and Butcher's Fifth Order Runge-Kutta Methods with Third Order Initial Value Problem (IVP),” Appl. Comput. Math., vol. 6, no. 6, p. 243, 2017, doi: 10.11648/j.acm.20170606.12. 
[5] A. B. M. Hame, I. Yuosif, I. A. Alrhama, and I. San, "Open Access The Accuracy of Euler and modified Euler Technique for First Order Ordinary Differential Equations with initial condition,” no. 9, pp. 334-338, 2017.

[6] H. Y. Lee, "Calculation of global error for initial value problem of ordinary differential equations," Int. J. Comput. Math., vol. 74, no. 2, pp. 237-245, 2000, doi: 10.1080/00207160008804937.

[7] O. Abraham, "Improving the Modified Euler Method," Leonardo J. Sci., vol. 6, no. 10, pp. 1-8, 2007.

[8] N. M. M. Yusop, M. K. Hasan, and M. Rahmat, "Comparison New Algorithm Modified Euler in Ordinary Differential Equation Using Scilab Programming,” Lect. Notes Softw. Eng., vol. 3, no. 3, pp. 199-202, 2015, doi: 10.7763/lnse.2015.v3.190.

[9] M. H. Afshar and M. Rohani, "Embedded modified Euler method: An efficient and accurate model," Proc. Inst. Civ. Eng. Water Manag., vol. 162, no. 3, pp. 199-209, 2009, doi: 10.1680/wama.2009.162.3.199.

[10] G. D. Hahn, "A modified Euler method for dynamic analyses," Int. J. Numer. Methods Eng., vol. 32, no. 5, pp. 943-955, 1991, doi: 10.1002/nme.1620320502.

[11] N. Samsudin, N. M. M. Yusop, S. Fahmy, and A. S. N. binti Mokhtar, "Cube arithmetic: Improving euler method for ordinary differential equation using cube mean," Indones. J. Electr. Eng. Comput. Sci., vol. 11, no. 3, pp. 1109-1113, 2018, doi: 10.11591/ijeecs.v11.i3.pp1109-1113.

[12] Burden, R. L., \& Faires, J. D. (1997). Numerical Analysis, Brooks. Cole, Belmont, CA.

\section{Authors' Profiles}

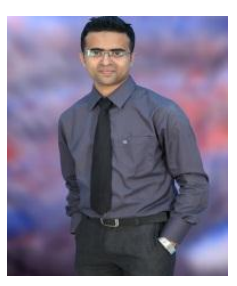

Mohammad Asif Arefin works at the Jashore University of Science and Technology (JUST), Jashore-7408, Bangladesh as a Lecturer in the Department of Mathematics. He obtained his B.Sc. (Honors) degree in Mathematics and M.S (Thesis) degree in Mathematics from Shahjalal University of Science and Technology (SUST), Sylhet-3114, Bangladesh. Later, he earned a Post Graduate Diploma (PGD) in Information Technology (IT) from the Institute of Information and Communication Technology (IICT), SUST. His research interest consists of Numerical Simulation, Mathematical Modeling, Meteorology, Solution of Partial Differential Equations, etc. He has good command over MATLAB, Maple, C, FORTRAN Programming Language, etc. His long-term goal is to be a successful person through teaching and research.

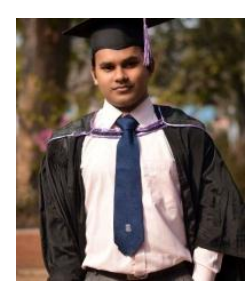

Nazrul Islam completed his B.Sc. (Honor's) in Mathematics from the University of Dhaka. He obtained his M.Sc. (Master's) in Applied Mathematics from the same university. He is currently working as a lecturer in the Department of Mathematics, Jashore University of Science and Technology, Jashore-7408, Bangladesh. His research interests are applied mathematics, numerical solution of ODE, PDE and spline approximations.

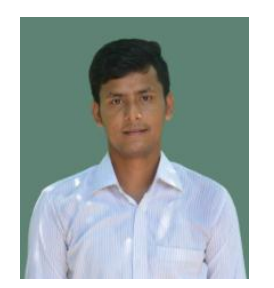

Biswajit Gain is currently studying at M.S in the department of Mathematics, Jashore University of Science \& Technology (JUST). He completed his B.Sc. (Honors) degree in Mathematics from Jashore University of Science \& Technology, Jashore-7408, Bangladesh. His research interest consists of Numerical solutions, Mathematical Modeling, Linear Algebra, Meteorology, etc. He is good at MATLAB, C, and Fortran programming language.

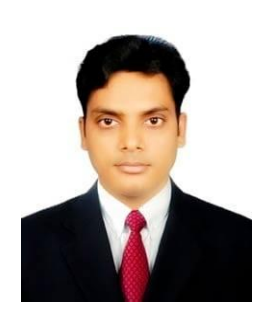

Md. Roknujjaman is a Ph.D. student at Tsukuba University, Japan under MEXT Scholarship. He is from Bangladesh. He doing his research in the field of fluid Engineering. Basically, his research area is biofluid mechanics and computational fluid dynamics. His long time goal is to be a good Researcher.

How to cite this paper: Mohammad Asif Arefin, Nazrul Islam, Biswajit Gain, Md. Roknujjaman," Accuracy Analysis for the Solution of Initial Value Problem of ODEs Using Modified Euler Method ", International Journal of Mathematical Sciences and Computing(IJMSC), Vol.7, No.2, pp. 31-41, 2021. DOI: 10.5815/ijmsc.2021.02.04 